\title{
ANÁLISE DA DEMÊNCIA EM IDOSOS ADSCRITOS NA ESTRATÉGIA DE SAÚDE DA FAMÍLIA DO BAIRRO BOM PASTOR
}

\author{
ALANE TORRES DE ARAÚJO LIMA 1, ANNA CARLA SILVEIRA \\ RODRIGUES ${ }^{2}$, BRUNA TEIXEIRA RIBEIRO ${ }^{3}$, KARINA GAMA DOS \\ SANTOS SALES ${ }^{4}$.
}

\begin{abstract}
1 Graduanda de Medicina no Centro Universitário UNIFACIG. alane_torres@ hotmail.com.
2 Graduanda de Medicina no Centro Universitário UNIFACIG. ana11.gbi@hotmail.com.

3 Graduanda de Medicina no Centro Universitário UNIFACIG. bruna_teixeira12@ hotmail.com.

4 Mestre em Políticas Públicas e Desenvolvimento local. Secretária Municipal de Saúde de Manhuaçu e professora dos Cursos de Medicina e Enfermagem do Centro Universitário UNIFACIG. karina.gamadossantos@gmail.com.
\end{abstract}

\section{RESUMO}

Nas últimas décadas, devido à transição demográfica e epidemiológica, foi evidenciado o envelhecimento populacional e o aumento da prevalência das doenças crônicodegenerativas como a demência na população com mais de 60 anos. O processo de envelhecimento de grande parte da população vem acompanhado de declínios cognitivos, sendo imprescindível a identificação do problema na comunidade e a promoção de ações efetivas na sua prevenção, a fim de promover melhorias na qualidade de vida dessa população. Portanto, este artigo teve como objetivo analisar a capacidade cognitiva da população geriátrica adscrita na ESF Bom Pastor em uma cidade da Zona da Mata Mineira. Para isso, foram aplicados o Mini Exame do Estado Mental (MEEM) e a Escala de Depressão Geriátrica (GDS) em 79 idosos, destes 39 apresentaram algum grau de demência e 26 algum grau de depressão. Dos participantes com demência, 25 possuíam comorbidades sistêmicas crônicas e 17 apresentavam depressão. Já no grupo dos que não apresentavam demência (40 pacientes), 9 tinham depressão, o que denota um Risco Relativo (RR) de 1,57. Em relação ao sexo, a incidência de demência foi de $51 \%$ nas mulheres (RR 1.08) e de $46 \%$ nos homens (RR 0.91), demonstrando ser o sexo feminino fator de risco e o sexo masculino fator de proteção para a demência. Os RR's de demência para os graus de escolaridade analfabetos, 1-4 anos, 5-8 anos e acima de 8 anos foram respectivamente 2,09, 1, 0,9 e 0,38 , denotando que, quanto maior o nível de escolaridade, menor o risco de desenvolver algum grau de demência. De acordo com os resultados, têm-se que algumas variáveis denotam fator de risco, enquanto outras parecem ser protetoras. Alguns desses aspectos são constantes e não passíveis de alteração; entretanto, outros como escolaridade, comorbidades e depressão podem ser prevenidos e trabalhados a fim de reduzir essa expectativa maléfica à qualidade de vida do idoso.

Palavras-chave: Demência; Depressão; Idosos. 


\title{
ANALYSIS OF DEMENTIA IN ELDERLY ADSCRIBED IN THE FAMILY HEALTH STRATEGY OF BOM PASTOR NEIGHBORHOOD IN MANHUAÇU- MG
}

\begin{abstract}
In the last decades, due to the demographic and epidemiological transition, an aging population and an increase in the prevalence of chronic-degenerative diseases such as dementia in the population over 60 years of age have been evidenced. The aging process of a large part of the population has been accompanied by cognitive decline, and it is essential to identify the problem in the community and to promote effective actions in its prevention, in order to promote improvements in the quality of life of this population. Therefore, this article aimed to analyze the cognitive capacity of the geriatric population enrolled in the ESF Bom Pastor. For this, the Mini Mental State Examination (MMSE) and the Geriatric Depression Scale (GDS) were applied to 79 elderly people, of these 39 had some degree of dementia and 26 some degree of depression. Of the participants with dementia, 25 had chronic systemic comorbidities and 17 had depression. In the group of those who did not have dementia (40 patients), 9 had depression, which denotes a Relative Risk (RR) of 1.57. Regarding gender, the incidence of dementia was $51 \%$ in women (RR 1.08) and $46 \%$ in men (RR 0.91), demonstrating that the female sex is a risk factor and the male sex is a protective factor for dementia. The RRs of dementia for illiterate levels of education, 1-4 years, 5-8 years and above 8 years were 2.09, 1, 0.9 and 0.38, respectively, showing that the higher the level of education, the lower the risk of developing some degree of dementia. According to the results, it appears that some variables denote a risk factor, while others appear to be protective. Some of these aspects are constant and not subject to change, however, others, such as education, comorbidities and depression, can be prevented and addressed in order to reduce this harmful expectation to the quality of life of the elderly.
\end{abstract}

Keywords: Dementia; Depression; Elderly.

\section{INTRODUÇÃO}

Nas últimas décadas, ocorreu, no Brasil, uma mudança do perfil demográfico, sendo que o envelhecimento populacional marca essa nova fase. Além do mais, as doenças crônicas degenerativas vêm substituindo o antigo padrão de prevalência de doenças infecciosas (OLIVEIRA, 2016).

A alteração no padrão de doenças traz novas consequências para a população, em especial a população em constante crescimento que são os idosos. Dados evidenciam que aproximadamente $95 \%$ dos idosos com idade entre 60 a 75 anos possuem ao menos uma doença crônica. As doenças crônicas, por sua vez, encontram-se correlacionadas com a perda cognitiva (NORDON, 2009).

No que tange os aspectos do declínio de cognição e demências, estudos demonstram que as atividades diárias e do cotidiano tem sido afetadas pelos quadros 
anteriormente citados. Para evitar prejuízos futuros na qualidade de vida, destacam-se testes que auxiliam no diagnóstico do declínio cognitivo, dentre eles podemos evidenciar o Mini Exame do Estado Mental (MEEM), que é amplamente usado e identifica determinadas deteriorações da cognição (BUSTAMANTE, 2003; CHARCHAT-FICHMAN, 2005; MOTA, 2008). Graus variados de declínio da cognição podem afetar de maneira impactante e negativa a qualidade de vida dos idosos (OLIVEIRA, 2016).

A atenção primária consegue captar através de artifícios como o MEEM fatores de risco que estão intimamente relacionados ao declínio da cognição. E através de uma resposta multidisciplinar é possível oferecer uma melhora na qualidade de vida tal como minimizar fatores que predispõem ou agravam um quadro demencial (GARCIA; RODRIGUES, 2012).

Diante do exposto, este trabalho tem por finalidade demonstrar os resultados de um estudo realizado na Estratégia de Saúde da Família do Bairro Bom Pastor, em que foram avaliados os quadros de demência na população idosa comparando com os fatores de risco para a ocorrência.

\section{MATERIAIS E MÉTODOS}

Trata-se de um estudo observacional, transversal, realizado com a população idosa adscrita na área de abrangência da Estratégia de Saúde da Família do bairro Bom Pastor em Manhuaçu, Minas Gerais. Para a seleção dos participantes, foi analisada a listagem de pacientes da ESF, tendo como critério de inclusão ter 60 anos ou mais. Após isso, foram selecionados, de maneira aleatória, alguns destes idosos, de modo que, para cada micro-área de abrangência, houvesse pelo menos um paciente selecionado.

O número (n) de participantes do estudo foi 79 idosos, aos quais foram aplicados o Mini Exame do Estado Mental (MEEM) e a Escala de Depressão Geriátrica (GDS).

O MEEM é estruturado de forma a avaliar as funções cognitivas: memória, orientação, atenção, capacidade de nomeação e de obediência a comandos escritos e verbais, de redação livre de uma sentença e de cópia de um desenho poligonal. Trata-se de um teste breve de rastreio cognitivo para identificação de demência. A pontuação máxima é de 30 pontos. Como o resultado pode ser influenciado pelo nível de 
escolaridade, atribuem-se pontos de corte distintos de acordo com a escolaridade do paciente (analfabetos: 13 pontos, 5-8 anos de estudo: 18 pontos, >8 anos de estudo: 24 pontos (BRUCKI et al., 2003; SANTANA, 2016).

A GDS corresponde a um questionário com 15 perguntas acerca do estado de humor do entrevistado e de como este vê a sua vida. As respostas para as perguntas serão sempre "sim" ou "não, assim, para cada "sim", soma-se 1 ponto e para cada "não" a pontuação é 0. Ao final, da entrevista, avalia-se o resultado obtido de acordo com o escore de corte, o qual, se $\geq 5$ determina a presença de sintomas depressivos nos idosos (PARADELA, 2005).

Para a análise dos resultados, foram calculadas as taxas de incidência de demência na população do estudo, bem como as incidências de algumas variáveis (grau de escolaridade, sexo, presença de depressão, diabetes e hipertensão arterial sistêmica) na população com e sem demência. Por fim, foram calculados os riscos relativos para cada agravo em relação à exposição à demência, com o intuito de verificar quais agravos constituíram fator de risco para a demência na população do estudo.

\section{DISCUSSÃO DOS RESULTADOS}

Este estudo foi realizado com um total de 79 idosos, dentre os quais 39 (49\%) apresentaram algum grau de demência, 47 eram do sexo feminino, 32 do sexo masculino, 26 apresentaram algum grau de depressão. Em relação à escolaridade, 10 idosos eram analfabetos, 44 possuíam 1-4 anos de estudo, 11 frequentaram a escola por 5 - 8 anos e 14 usufruíram mais de 8 anos de escolaridade.

Dos 39 participantes que apresentavam demência em algum grau, 25 possuíam alguma comorbidade sistêmica crônica (Diabetes ou Hipertensão Arterial), enquanto que, dos 40 idosos que não apresentavam nenhum grau de demência, 30 possuíam alguma comorbidade.

Ao se analisar o risco entre a presença concomitante de demência e comorbidades sistêmicas crônicas, foi encontrado um Risco Relativo (RR) de 0,77 possuir alguma comorbidade, assim não foi considerado fator de risco para se ter demência. Entretanto, em estudo caso-controle realizado na Universidade Federal de Minas Gerais (UFMG), ao se analisar os grupos, o oddsratio encontrado em relação a 
possuir comorbidades e ter demência foi de 6,2, evidenciando que ter comorbidade é considerado fator de risco para demência.

O envelhecer traz consigo alterações na mobimortalidade. As doenças crônicas degenerativas passam a impactar na vida dos idosos cursando com graus variados de dependência e fragilidade ocasionadas, em especial, pelos quadros demenciais. A prevalência de comorbidades é maior nos idosos com demência, afetando de modo significativo o declínio funcional e a cognição (PIMENTA et al., 2013).

Em um estudo realizado no serviço de Geriatria, ao se analisar quadros de demência e depressão, constava que $95 \%$ dos idosos eram portadores de alguma doença crônica, sendo que o maior número $(62,2 \%)$ foi encontrado em portadores de hipertensão arterial sistêmica. Ademais, o autor ressalta que é acreditável que ocorra um subdiagnóstico ou um mau tratamento de determinadas comorbidades, entre elas hipertensão arterial sistêmica e diabetes mellitus, devido à dificuldade em queixar-se que os idosos possuem e em virtude da cognição prejudicada causando uma baixa adesão e entendimento terapêutico (PIMENTA et al., 2013).

A importância de controlar quadros de hipertensão arterial sistêmica se dá em razão de que sua falha terapêutica está ligada de maneira direta no declínio da cognição e das habilidades no decorrer do tempo. Testes e estudos aplicados em idosos evidenciam que, naqueles com hipertensão, o desempenho de funções cognitivas é menor em comparação aos não hipertensos. Algumas alterações mais citadas são: declino da memória verbal e visual, declínio em testes de inteligência e de atividades psicomotoras (ZASLAVSKY; GUS, 2002).

Igualmente, há evidências de que a diabetes mellitus e a hipertensão arterial sistêmica estão associadas a demências subcorticais, comprometimento de memória, disfunção executiva, alterações frontais, declínio da atenção, sintomas depressivos, motor lentificado e até mesmo sintomas de Parkinson (PIMENTA, 2011).

Em se tratando da demência vascular, dados comprovam que portadores de diabetes mellitus têm uma maior prevalência dessa demência se comparados com portadores de outras comorbidades. (PIMENTA, 2011)

Ao relacionar a presença de distúrbios depressivos e demência, foi observado que, dos 39 pacientes com demência, 17 ( 44\%) apresentavam também depressão. Já no grupo dos que não apresentavam demência (40 pacientes), apenas 9 (22,5\%) tinham 
depressão. Calculando-se o risco relativo de distúrbios depressivos nos pacientes com demência, chegamos ao resultado de 1,57, o que configura a depressão como um fator de risco para a demência. Porém, a depressão, por si só, produz déficits de memória, sobretudo a partir dos 40 anos de idade, podendo ser confundida com um estágio inicial de demência, o que pode gerar viéses quando se compara as duas comorbidades (CARNEIRO, 2016).

A literatura acerca do assunto expressa a existência de uma linha tênue entre a depressão e a demência. A depressão é reconhecida como um fator de risco independente para uma posterior demência ou como um estado prodrômico, que antecede o início da degradação cognitiva (FORLENZA et al., 2010; TOBE, 2012, RAMOS, 2014).

No que concerne ao sexo, dentre os pacientes com demência, 24 eram do sexo feminino e 15 do sexo masculino. No grupo sem demência, 23 eram do sexo feminino e 17 do sexo masculino. Assim, dentre as mulheres, a incidência de demência foi de $51 \%$ e entre os homens de $46 \%$. Esses resultados demonstraram que o sexo feminino apresenta um RR de 1.08para demência, enquanto que ser do sexo masculino agrega um RR de0.91, o que neste estudo, demonstrou o sexo feminino ser fator de risco, e o sexo masculino ser fator de proteção para a demência. Tal resultado está em concordância ao demonstrado por Lentsck et al. (2015), estudo no qual os sinais de demência tiveram uma associação positiva para o sexo feminino $(\mathrm{RP}=1,8)$. Adicionalmente, Burlá et al. (2013), em revisão sistemática da literatura acerca das prevalências de demência no Brasil, demonstrou que, em todas as áreas estudadas, a prevalência de demência foi maior entre as mulheres.

Já em relação a escolaridade, foi observado neste estudo que dos 39 pacientes que apresentaram algum grau de demência, 9 eram analfabetos, 22 estudaram apenas por 1-4 anos, 5 frequentaram escola por 5-8 anos e apenas 3 dos dementes tinham escolaridade maior que 8 anos. Dentre os pacientes sem demência, apenas 1 era analfabeto, 22 estudaram por 1-4 anos, 6 frequentaram escola por 5-8 anos e 11 possuíam escolaridade acima de 8 anos (BURLÁ et al, 2013).

Ao calcularmos o risco relativo, nesse estudo, do analfabetismo com a demência chegamos a o valor de 2,09 configurando se assim o fato de não ser alfabetizado como um alto fator de risco para demência. O risco relativo entre a escolaridade por 1-4 anos 
e a demência, por sua vez, neste estudo foi de 1, ou seja, não existe associação entre esse grau de escolaridade e algum nível de demência.

No tocante ao grau de escolaridade por 5-8 anos e a demência, o risco relativo obtido através dos dados coletados foi de 0,9, demonstrando que esse grau de escolaridade é um fator de proteção contra a demência. Isso foi percebido também entre a escolaridade acima de 8 anos e a demência, que também se configura um fator de proteção, uma vez que o resultado do risco relativo encontrado foi de 0,38 .

Logo, percebe-se claramente que, quanto maior o nível de escolaridade do paciente, menor o risco de ele desenvolver algum grau de demência. Os resultados encontrados neste estudo estão em concordância com Bickel e Kurz (2009) que, no seu estudo, encontraram uma forte associação entre o diagnóstico de demência e o baixo nível educacional. Machado et al. (2011), através de um estudo realizado com 74 idosos, demonstraram que aqueles com 1 ano de estudo ou menos, tem 3,86 vezes mais chances de ter algum grau de declínio cognitivo dos que apresentaram uma escolaridade acima de 5 anos. $\mathrm{O}$ resultado encontrado reafirma o obtido neste estudo, que, quanto menor o nível de escolaridade do paciente, maior o risco deste desenvolver demência.

Por outro lado, Brito-Maques e Cabral-Filho, num estudo realizado em 2005, identificaram que ter mais de oito anos de escolaridade é fator protetivo contra redução das habilidades para solução de testes cognitivos em sua amostra. Tal resultado está em concordância com o encontrado no nosso estudo, em que o risco relativo entre escolaridade acima de 8 anos e a demência foi menor que 1, ou seja, é um fator de proteção em relação ao declínio cognitivo.

\section{CONCLUSÃO}

Ao realizar este trabalho, foi percebido a importância de se avaliar e pesquisar fatores relacionados ao envelhecimento e a demência a fim de criar uma forma de cuidado a futura população idosa, bem como prevenir agravos cognitivos inerentes à população idosa atual. Através do processo de envelhecimento, observam-se mudanças na competência das habilidades cognitivas, apesar de que tais prejuízos não necessariamente afetam o cotidiano dos idosos e seus familiares. 
Além disso, a demência é uma variável importante a ser investigada no paciente, uma vez que se encontra associada a uma série de prejuízos como instabilidade, depressão, entre outros fatores que podem, por sua vez, afetar a qualidade de vida do idoso.

Vale ressaltar ainda que, com este estudo, foram analisados alguns aspectos que podem ser fator de risco ou de proteção para o desenvolvimento de demência na amostra colhida. De acordo com os resultados, ficou claro que algumas dessas variáveis que são fator de risco, como o sexo por exemplo são constantes e não estão passíveis de melhora, ou mudança do aspecto. Entretanto, outros fatores como escolaridade, comorbidades e a depressão podem ser devidamente prevenidos e trabalhados a fim de reduzir essa expectativa maléfica à qualidade de vida do idoso.

Portanto, está explicito que a saúde integral do idoso é de suma importância, tornando essas condições de grande relevância, visto que elas estão ligadas de maneira intrínseca a quadros demências e, consequentemente, a declínios cognitivos, prejudicando de modo fundamental na qualidade de vida dos idosos.

\section{REFERÊNCIAS}

BICKEL, H.; KURZ, A. Educação, ocupação e demência: o estudo das irmãs da escola da Baviera. Distúrbios cognitivos geriátricos por demência, v. 27, n.6, p. 548-546, 2009.

BRITO-MARQUES, P. R.; CABRAL-FILHO, J. E. Influência da idade e escolaridade no desempenho em uma versão modificada do Mini-Mental: um estudo no nordeste do Brasil. Arquivos de Neuro-Psiquiatria, v. 63, n.3, p. 583-587, 2005.

BRUCKI, S.M.D. et al. Sugestões para o uso do mini-exame do estado mental no Brasil. Arquivos de Neuro-Psiquiatria, v. 61, n. 3B, p. 777-781, 2003.

BURLÁ, C. et al. Panorama prospectivo das demências no Brasil: um enfoque demográfico. Ciência \& Saúde Coletiva, v. 18, n. 10, p. 2949-2956, 2013.

BUSTAMANTE, S. E. Z. et al. Instrumentos combinados na avaliação de demência em idosos: resultados preliminares. Arquivos de Neuro-Psiquiatria, v. 61, n. 3A, p. 601-6, 2003.

CARNEIRO, J. P.; CABRAL, H. A linha ténue entre a demência e depressão no idoso: relato de caso. Revista Portuguesa de Medicina Geral e Familiar, v. 32, n. 2, p. 118$124,2016$. 
CHARCHAT-FICHMAN, F. H. et al. Declínio da capacidade cognitiva durante o envelhecimento. Revista Brasileira de Psiquiatria, v. 27, n. 21, p. 79-82, 2005.

GARCIA, M.A.A. et al. O envelhecimento e a saúde. Revista de Ciências Médicas, v. 13, n. 2, p. 221-231, 2002.

LENTSCK, M. H. et al. Prevalência de sintomas depressivos e sinais de demência em idosos na comunidade. Revista Eletrônica de Enfermagem. v. 17, n. 3, p. 1-9, 2015.

MOTA, M. M. P. E. et al. Triagem cognitiva: comparações entre o mini-mental e o teste de trilhas. Estudos de Psicologia Campinas, v. 25, n. 3, p. 353-359, 2008.

MACHADO, J. C. et al. Declínio cognitivo de idosos e sua associação com fatores epidemiológicos em Viçosa, Minas Gerais. Revista Brasileira de Geriatria e Gerontologia, v.14, n. 2, p. 109-121, 2011.

NORDON, D. G., et al. Perda cognitiva em idosos. Revista da Faculdade de Ciências Médicas de Sorocaba, v. 11, n. 3, p. 5-8, 2009.

OLIVEIRA, A. T. R. Envelhecimento populacional e políticas públicas: desafios para o Brasil no século XXI. Revista Brasileira de Geografia Econômica, São Gonçalo, 2016. Disponível em: http://journals.openedition.org/espacoeconomia/2140. Acesso em: 30 mar. 2019.

PARADELA, E.M.P. et al. Validação da escala de depressão geriátrica em um ambulatório geral. Revista de Saúde Pública. v. 39, n.6, p. 918-923, 2005.

PIMENTA, F.A.P et al. Doenças crônicas, cognição, declínio funcional e Índice de Charlson em idosos com demência. Revista da Associação Médica Brasileira. v.59 n.4, p. 326-334, 2013.

PIMENTA, F.A.P. Fatores Relacionados ao Perfil Clínico, Funcional, Cognitivo, Genético e de Predição da Mortalidade em Pacientes Idosos com Depressão e Demência. Belo Horizonte, 2011. Disponível em: <http://bvsms.saude.gov.br/bvs/publicacoes/premio2011/doutorado/doutorado_Fausto_ Aloisio.pdf $>$. Acesso em: 30 mar. 2019.

RAMOS, T. S. Depressão e Demência no Idoso: diagnóstico diferencial e correlações. Porto, 2014. Disponível em: https://scholar.google.com.br/scholar?q=Depress\%C3\%A3o+e+Dem\%C3\%AAncia+no +Idoso:+diagn\%C3\%B3stico+diferencial+e+correla\%C3\% A7\%C3\%B5es.\&hl=ptBR\&as_sdt=0\&as_vis=1\&oi=scholart $>$. Acesso em: 30 mar. 2019.

SANTANA, I. et al. Mini-Mental State Examination: Avaliação dos Novos Dados Normativos no Rastreio e Diagnóstico do Déficit Cognitivo. Acta Médica Portuguesa, v. 29, n. 4, p. 240-248, 2016.

ZASLAVSKY, C.; GUS, I. Idoso. Doença Cardíaca e Comorbidades. Arquivos Brasileiros de Cardiologia. v.79, n.6, p. 635-639, 2002. 